\title{
Comparison between Levobupivacaine Alone and Levobupivacaine with Fentanyl in Extending the Duration and Postoperative Analgesia of Supraclavicular Brachial Plexus Block for Elective Upper Limb Orthopedic Surgeries \\ Hala Mahmoud, Fawzy Abbas, Mahmoud Gamal El-Din Youssef*, Ghada Abdelgaber \\ Department of Anesthesia and I.C.U and Pain Management, Faculty of Medicine, Sohag University, Egypt \\ *Corresponding author: Mahmoud Gamal El-Din, Mobile: (+20)1066117686, E-Mail: damong255@gmail.com
}

\begin{abstract}
Background: Brachial plexus Block is widely utilized nowadays in high risk patients with high safety margins providing hemodynamic stability and optimal muscle relaxation.

Objectives: The aim of the work was to evaluate the efficacy of fentanyl as an adjuvant on hemodynamic stability, onset and duration of the block and postoperative analgesia.

Patients and methods: This double blinded randomized study included a total of 100 patients subjected for upper limb orthopedic surgeries using supraclavicular brachial plexus block using levobupivacaine, attending at Sohag University Hospital. Patients were divided randomly into two groups, group L (control group) and group LF (study group). Regular hemodynamics monitoring were done.

Results: there was no significant difference between both groups as regard hemodynamics, although there was significant faster onset of the block and prolongation of its duration in group LF. Also, the VRS pain score was significantly lower in the postoperative period in group LF and less analgesic requirement.

Conclusion: It could be concluded that using fentanyl as an adjuvant to levobupivacaine is effective in enhancing the onset of the brachial plexus block and prolonging the duration of motor and sensory blocks with no hemodynamic changes. It effectively prolongs postoperative analgesia and lowers the analgesic requirements.
\end{abstract}

\section{INTRODUCTION}

Brachial plexus blocks provide a useful alternative to general anesthesia as they achieve near-ideal operating conditions by producing complete muscular relaxation, maintaining stable intra-operative hemodynamics and the risk associated with general anesthesia are avoided ${ }^{(\mathbf{1})}$.

Of various local anesthetics, Levobupivacaine is used most frequently, as it has a long duration of action varying from 3 to 8 hours and less cardiotoxic effect with intravascular spread which is the major risk factor for brachial plexus block ${ }^{(2)}$.

Various drugs were added to local anesthetics like neostigmine, opioids, dexamethasone, hyaluronidase, and clonidine to modify the block regarding producing quick onset of action, good quality of anesthesia, prolonged duration and effective post-operative analgesia ${ }^{(3)}$. The addition of opioids in brachial plexus block is reported to improve success rate and postoperative analgesia, by some authors whereas others have found no effect ${ }^{(4)}$.

Fentanyl is an opioid analgesic is known to produce antinociception and to enhance the effect of local anesthetics when given epidurally or intrathecally, studies showed that it could act directly on the peripheral nervous system. Primary afferent tissues (dorsal roots) have been found to contain opioidbinding sites. Due to the presence of bidirectional axonal transport of opioid-binding protein, fentanyl can penetrate the nerve membranes and act on the dorsal horn. This could also account for the prolonged analgesia ${ }^{(5)}$. However, fentanyl is reported to have a local anesthetic action ${ }^{(6)}$.

The aim of the current work was to study the effect of adding fentanyl to levobupivacaine as regard hemodynamics stability, onset and duration of the block, postoperative analgesia, and complications.

\section{PATIENTS AND METHODS}

This double blinded randomized study included a total of 100 patients aged between 18 and 60 years with ASA I and II score and posted for elbow, forearm, and hand orthopedic surgeries, attending at Sohag University Hospital. This study was conducted between.

\section{Ethical approval:}

Written informed consent of all the subjects was obtained.

Approval of the ethical committee of Sohag university was obtained.

All patients received anesthesia by supraclavicular brachial plexus block using levobupivacaine.

The included subjects were randomly divided into two groups; Group L (control group) consisted of 50 patients received $22.5 \mathrm{ml}$ levobupivacaine $0.5 \%+7.5 \mathrm{ml}$ normal saline and Group LF (study group) consisted of 50 patients received $22.5 \mathrm{ml}$ levobupivacaine $0.5 \%+$ $1 \mathrm{ml}$ Fentanyl $(50 \mu \mathrm{g})+6.5 \mathrm{ml}$ normal saline. Routine preoperative investigations as complete blood picture, renal function tests, liver function tests, and coagulation profile were done. 


\section{Intraoperative monitoring of the following:}

1. Hemodynamics: monitoring of HR, MBLP, RR and $\mathrm{SO} 2$ every 15 minutes till the end of surgery.

2. Onset of sensory block: the time between the full deposition of local anesthetic using pin prick test, this was checked at: $0,5,10,15$ and 20 minutes till loss of pinprick sensation.

3. Onset of motor block: this was checked at $0,5,10,15$ and 20 minutes using modified Bromage scale till complete loss of movement.

4. Duration of Sensory Block: which was checked every 30 minutes until regaining of pinprick sensation.

5. Duration of motor block: which was checked every 30 minutes until regain of movements.

\section{Postoperative monitoring of the following:}

1. Hemodynamics: monitoring of HR, MBLP, RR and $\mathrm{SO}_{2}$ every 1 hour for 6 hours postoperative.Pain assessment: using Verbal rating scale (VRS) every 30 minutes until 6 hours then every 1 hour until 12 hours postoperative.
2. Time of first analgesic request it was recorded at the time from the supraclavicular brachial plexus block administration to the patient's first request for analgesic medication, also the number of times patient requested analgesia was recorded

3. Adverse effects: possible complications such as local hematoma, hemothorax, pneumothorax, intravascular injection, nausea, vomiting, any signs of local anesthetic toxicity and hallucinations.

\section{RESULTS}

There was no significant difference regarding demographic data (age, sex, and weight) or intraoperative duration between both groups.

As regard hemodynamics, there is no significant difference in heart rate measurements in all times monitored in the study. Although HR measurements of group LF were lower than group L but no significant difference.

There was no significant difference between both groups regarding MBLP, $\mathrm{RR}$ or $\mathrm{SO}_{2}$.

Table (1): Demographic Data

\begin{tabular}{|l|l|c|c|c|}
\hline Demographic variable & & $\begin{array}{c}\text { Group L } \\
\mathbf{N = 5 0}\end{array}$ & $\begin{array}{c}\text { Group LF } \\
\mathbf{N = 5 0}\end{array}$ & \multirow{2}{*}{ P value } \\
\hline Age (years) & Mean \pm SD) & $35.48 \pm 12.04$ & $32.68 \pm 10.2$ & 0.2126 (N.S) \\
\hline \multirow{2}{*}{ Sex } & Male no (\%) & $33(66 \%)$ & $32(64 \%)$ & 0.823 (N.S) \\
\cline { 2 - 5 } & Female no (\%) & $17(34 \%)$ & $18(36 \%)$ & \\
\hline Weight (Kg) & Mean \pm SD & $67.8 \pm 7.23$ & $70.58 \pm 5.46$ & 0.325 (N.S) \\
\hline Duration (Min) & Mean \pm SD & $87.6 \pm 20.757$ & $86.5 \pm 22.61$ & 0.8004 (N.S) \\
\hline
\end{tabular}

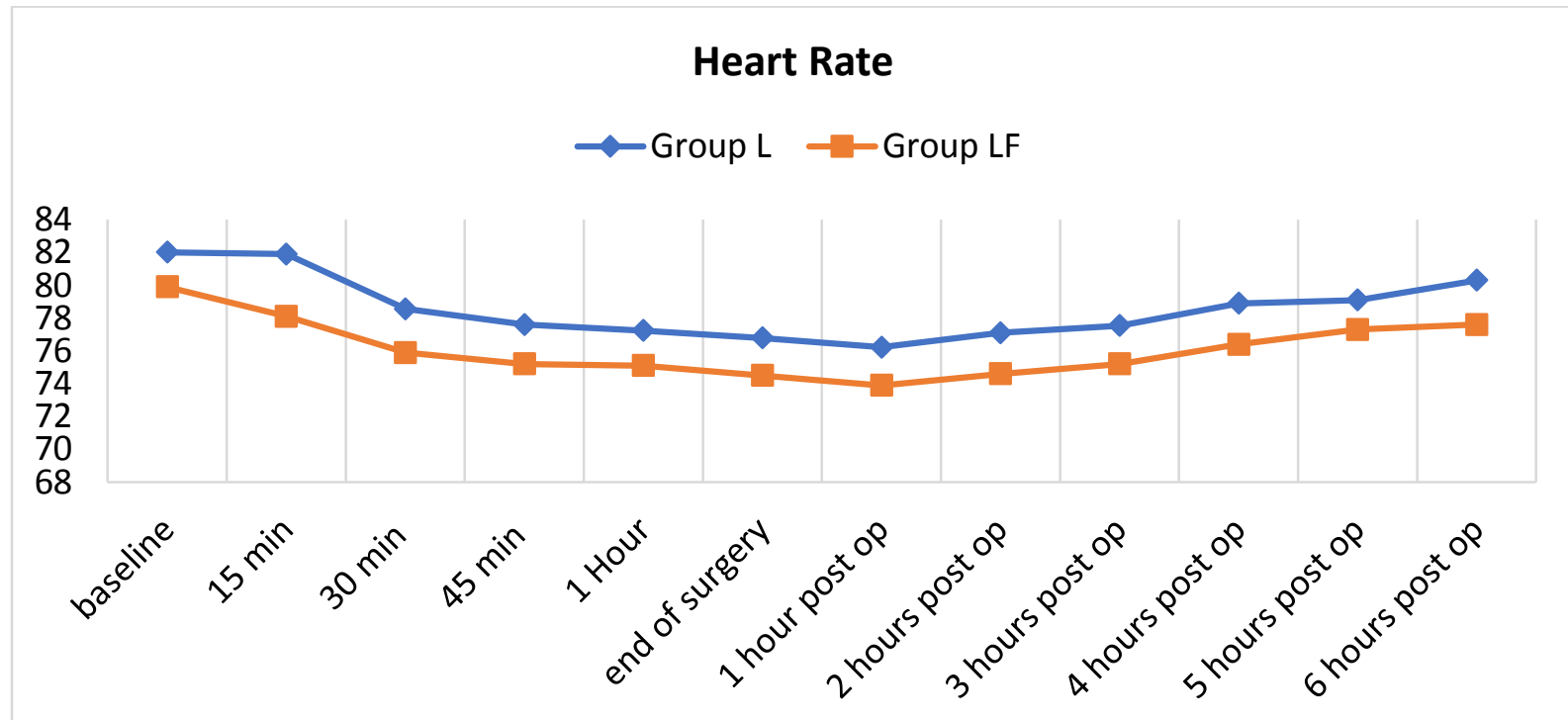

Fig. (1): Means of heart rate of both groups at set times.

As regard onset of sensory block, there was significant difference between the two studied groups, the sensory onset was significantly faster in group LF $(10.8 \pm 3.6 \mathrm{~min})$ compared to group L $(15.3 \pm 5.8 \mathrm{~min})$.

As regard onset of motor block, there was a significant difference between the two study groups, it was faster in group LF (12.7 $\pm 3.5 \mathrm{~min})$ compared to group L (17.4 $\pm 4.3 \mathrm{~min})$. Regarding duration of sensory block, there was a highly significant difference between the two study groups $(\mathrm{P}<0.0001)$. It was highly significant longer in group LF $(658.4 \pm 14.08 \mathrm{~min})$ compared to group L (524.8 $\pm 12.7 \mathrm{~min})$.

As for duration of motor block, there was a highly significant difference between the two study groups, it was highly significant longer in group LF (549.8 $\pm 40.8 \mathrm{~min})$ compared with group L (463.2 $\pm 12.2 \mathrm{~min})$. 


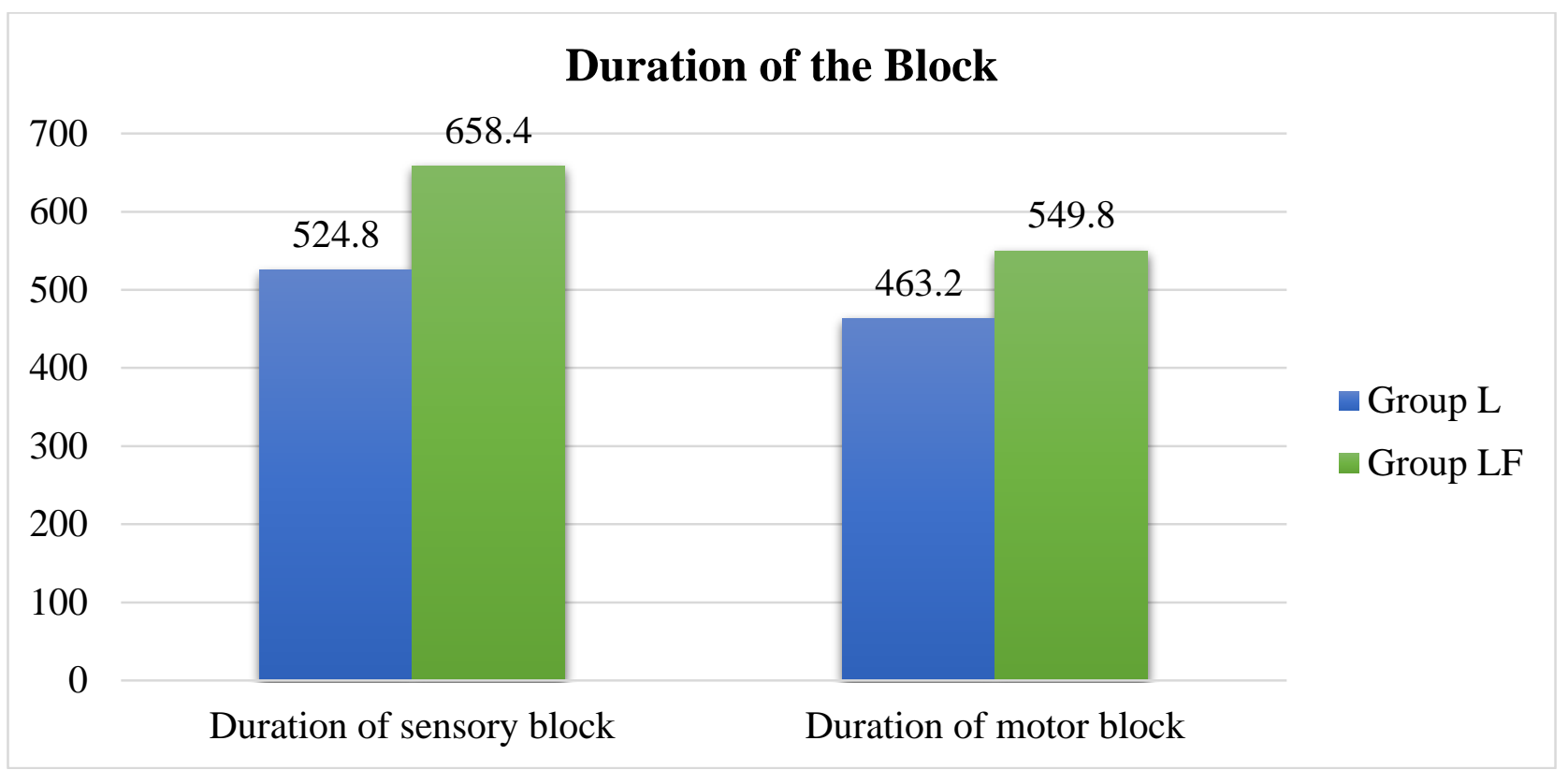

Fig. (2): Mean duration of motor and sensory blocks.

On comparing the VRS of the two study groups postoperative every 1 hour for 24 hours, there was a highly significant difference in VRS between the two studied groups at all times studied, VRS was lower in group LF than group L.

Comparing the patient request for rescue analgesia, in Group L only 11 patients did not ask for analgesia, although in group LF, 29 patients didn't ask for rescue analgesia which shows significant difference between both groups.

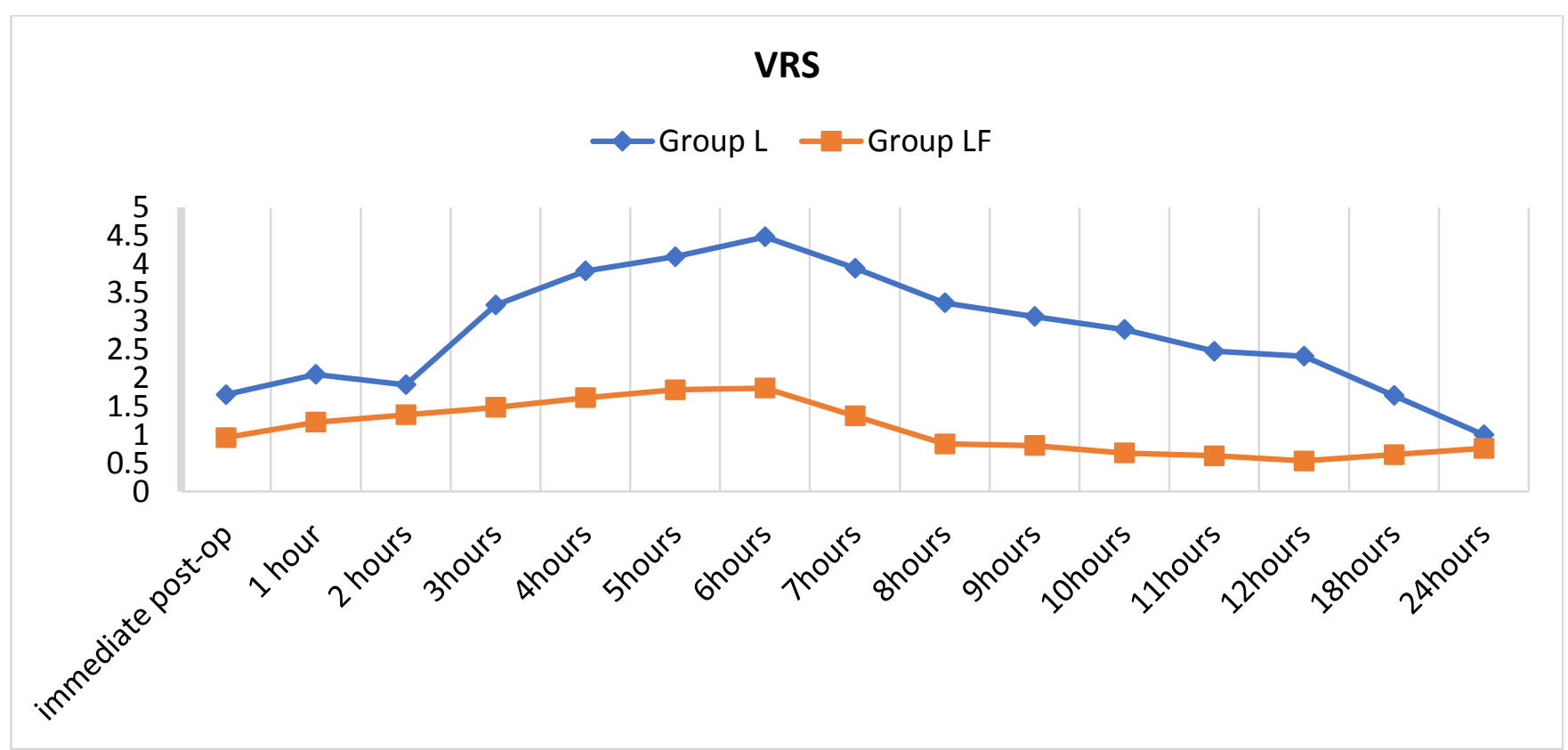

Fig. (3): Mean VRS score of both groups at set times.

Also, on comparing the first time the patients ask for analgesic postoperative after how many minutes, there was earlier inquiry in Group L (Mean 279.7) than Group LF (Mean 372.8).

On comparing these values, there's highly significant difference indicating earlier inquiry for group L. 


\section{No of analgesic inquiry}

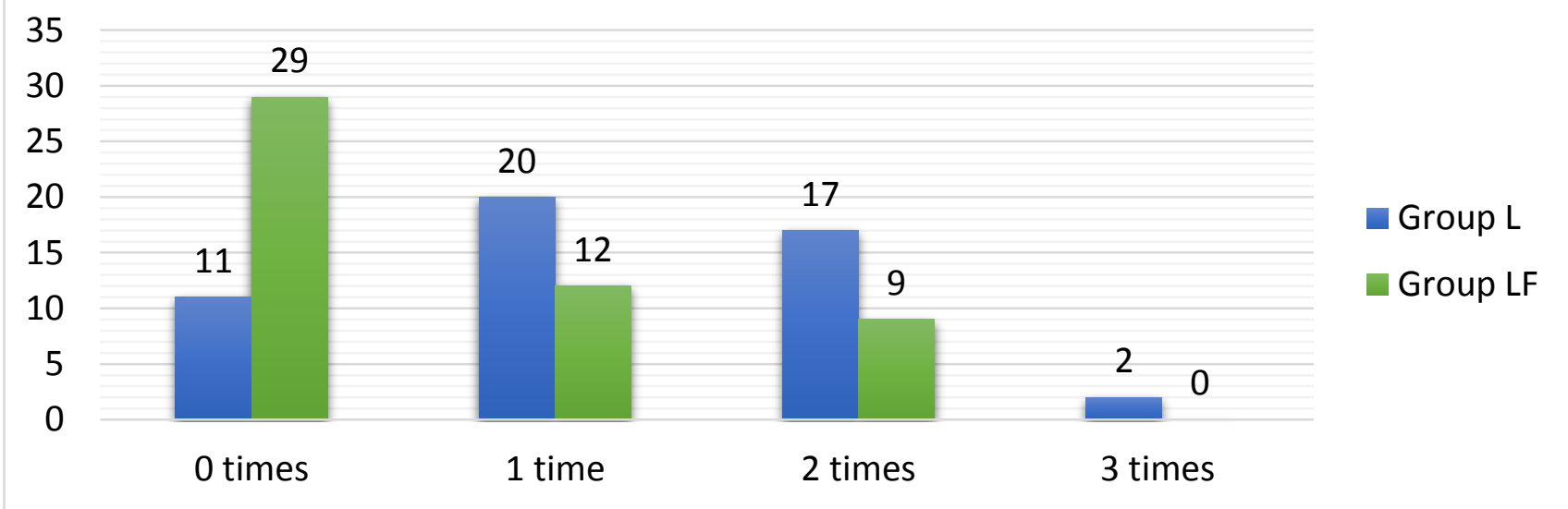

Fig. (4): Comparison between number of analgesic inquiries of both groups.

As regard complications, only one patient developed hypotension in Group LF, and no one suffered hypotension in Group L.

Also, nausea was noticed in two patients in the same group (Group LF) compared to one patient in Group L, bradycardia was noticed once in Group LF in the same patient who developed hypotension, also in one patient in Group L.

Table (2): Complications frequency and percentage.

\begin{tabular}{|l|l|l|l|l|}
\hline & \multicolumn{2}{|c|}{ Group L (N=50) } & \multicolumn{2}{c|}{ Group LF (N=50) } \\
\hline & $\mathbf{N}$ & $\mathbf{\%}$ & $\mathbf{N}$ & $\mathbf{\%}$ \\
\hline Pneumothorax & 0 & 0 & 0 & 0 \\
\hline Hemothorax & 0 & 0 & 0 & 0 \\
\hline Nausea & 1 & $2 \%$ & 2 & $4 \%$ \\
\hline Vomiting & 0 & 0 & 0 & 0 \\
\hline Bradycardia & 1 & $2 \%$ & 1 & $2 \%$ \\
\hline Hypotension & 0 & 0 & 1 & $2 \%$ \\
\hline L.A toxicity & 0 & 0 & 0 & 0 \\
\hline
\end{tabular}

\section{DISCUSSION}

Brachial plexus block is an excellent alternative to general anesthesia for a wide variety of upper limb procedures ${ }^{(1)}$. In the current study and as regard hemodynamics monitoring perioperatively, both groups showed no significant difference of mean blood pressure when compared in both groups ( $\mathrm{p}$ value $=$ 0.129 ), while the mean heart rate measurements of group LF were lower than L group with no statically significant difference $(\mathrm{P}$ value $=0.325)$.

Our results came in agreement with Kumar et al. (7) in their study Comparing Fentanyl and Nalbuphine as an Adjuvant to $0.5 \%$ Bupivacaine showed that there's decline in heart rate and Mean blood pressure from its baseline at all periods of observation, but that decline by adding fentanyl rather than nalbuphine was not statically significant.

Also, these results came in agreement with Paluvadi and Sasha ${ }^{(8)}$ study on the effect of addition of fentanyl to xylocaine hydrochloride in supraclavicular brachial plexus block who showed that there is no significant difference regarding Heart rate and Mean blood pressure between the study group (xylocaine $1.5 \%+$ fentanyl) and control group (xylocaine $1.5 \%$ )

Also, our results came in agreement with Hamed et al. (9) study on effect of adding Fentanyl and dexmedetomidine as adjuvant to bupivacaine. They observed that there was no significant difference as regard heart rate and blood pressure between the fentanyl group and the control group.

Shiva and Kumar (10) showed in their comparative study between bupivacaine alone and bupivacaine with fentanyl in axillary plexus block also showed no significant difference regarding Heart rate and Blood pressure but still lower in patients receiving fentanyl with bupivacaine.

These results came in agreement with results of Farooq et al. ${ }^{(11)}$ in their double blind randomized study to evaluate the efficacy of fentanyl and dexmedetomidine as adjuvant to ropivacaine in brachial plexus block carried out on 60 patients, found that HR is lower with group receiving fentanyl than control group. They explained that may be due to the effect of fentanyl as a Vagus nerve mediated bradycardia. 
As regard onset of sensory and motor block in our study, there was significant difference between both groups $(\mathrm{P}>0.001)$, Group LF was faster onset of sensory loss (10.8 $\pm 3.6 \mathrm{~min})$ in comparison with Group $\mathrm{L}(15.3 \pm 5.8 \mathrm{~min})$ in sensory block. As for motor block Group LF was faster onset (12.7 $\pm 3.5 \mathrm{~min})$ than group $\mathrm{L}(17.4 \pm 4.3 \mathrm{~min})$, the onset of levobupivacaine with the same dose was 19.7 minutes for sensory block onset and 21.5 for motor onset ${ }^{(\mathbf{1 2})}$.

These results are in agreement with those of Hamed et al. ${ }^{(9)}$ study on effect of adding Fentanyl and dexmedetomidine as adjuvant to bupivacaine. It indicated that adding fentanyl to local anesthetic produces faster sensory onset $(11.8 \pm 3.4 \mathrm{~min})$ and motor onset $(13.7 \pm 2.4 \mathrm{~min})$ than control group sensory onset $(16.3 \pm 4.8 \mathrm{~min})$ and motor onset $(18.4 \pm 5.2 \mathrm{~min})$.

Also, our results came in agreement with Hembrom et al. ${ }^{(13)}$ study with faster sensory onset $(2.5 \pm 0.75 \mathrm{~min})$ and faster motor onset $(3.7 \pm 1.3 \mathrm{~min})$ by adding fentanyl compared to control group sensory onset $(4.5 \pm 1.5 \mathrm{~min})$ and motor onset $(7.5 \pm 1.9 \mathrm{~min})$. This is faster than the onset of our study and this could be due to the larger volume of levobupivacaine $0.5 \%$ (30c.c) and larger dose of fentanyl (100 $\mu \mathrm{g}$ ) used.

Our results also came in agreement with Chavan et al. ${ }^{(6)}$ in their study to find effect of adding fentanyl to local anesthetic (bupivacaine 0.5\%) in supraclavicular brachial plexus block carried out on 50 patients.

Regarding duration of the motor and sensory block in our study, there was statically significant difference between both groups. The duration of sensory block was much longer in patients receiving levobupivacaine with fentanyl mixture $(658.4 \pm 14 \mathrm{~min})$ than patients receiving levobupivacaine alone $(524.8 \pm 12.7 \mathrm{~min})$. Also, the same difference with the duration of motor block Group LF was longer duration (549.8 $\pm 40.8 \mathrm{~min})$ than group L (463.2 $\pm 12.2 \mathrm{~min})$.

Our results came in agreement with Hembrom $e t$ al. ${ }^{(13)}$, Hassan et al. ${ }^{(14)}$ Manohar et al. ${ }^{(15)}$ and Kumar et al. ${ }^{(16)}$ who observed a significant prolongation of the duration of sensory block and motor block in study groups (fentanyl with levobupivacaine) compared to control groups.

Shrestha et al. ${ }^{(17)}$ concluded in their study that currently available local anesthetics can provide analgesia for limited period of time when used as single injection. To extend the analgesia period beyond the operating rooms, various methods have been tried with the aim of prolonging the local anesthetic action, like use of different adjuvants with local anesthetics including epinephrine, clonidine, fentanyl, ketamine, midazolam and dexamethasone.

Rajkhowa et al. ${ }^{(4)}$ mentioned in their study by adding fentanyl as adjuvant in brachial plexus nerve block that the mechanism of fentanyl in prolonging analgesia may be caused by the presence of peripheral opioid receptors, but their existence in peripheral tissue is still doubtful.

They also explained in their study that fentanyl used with ropivacaine prolonged the duration of sensory and motor blockade probably by directly binding with opioid binding sites on the dorsal nerve roots aided with these axonal transports or by diffusing into surrounding tissues and subsequently into the epidural and subarachnoid spaces; it may also have been central opioid receptor mediated after systemic absorption of fentanyl.

Chavan et al. ${ }^{(6)}$ also explained the possible mechanisms of action for the improved analgesia produced by the peripheral application of fentanyl. They explained that fentanyl may potentiate local anesthetic action via central opioid receptor-mediated analgesia by peripheral uptake of fentanyl to systemic circulation.

Karakaya and Büyükgöz ${ }^{(18)}$ explained that fentanyl may diffuse from the brachial plexus sheath to epidural and subarachnoid spaces and then bind with the opioid receptor of the dorsal horn, but it is unclear that whether a sufficient dose of fentanyl diffused to the epidural or subarachnoid spaces to cause adequate analgesia.

Fields and Emson (19) also explained that fentanyl can act directly on the peripheral nervous system as the primary afferent tissues (dorsal roots) are found to contain opioid-binding sites. Due to the presence of bidirectional axonal transport of opioidbinding protein, fentanyl can penetrate the nerve membranes and act on the dorsal horn.

As regard postoperative analgesia in our study using verbal rating scale (VRS), there was statically high significant difference between both groups $(\mathrm{P}$ value $<0.0001)$. Patients who received mixture of levobupivacaine with fentanyl (group LF) showed lower VRS score $(1.35,1.65,1.82,0.84,0.68,0.54)$ at ( 2 h, 4 h, 6 h, 8 h, $10 \mathrm{~h}$ and 12 hours), respectively. And patients of who received Levobupivacaine only showed VRS score $(1.88,3.88,4.48,3.32,2.85,2.38)$ at $(2 \mathrm{~h}, 4$ $\mathrm{h}, 6 \mathrm{~h}, 8 \mathrm{~h}, 10 \mathrm{~h}$ and 12 hours), respectively. This indicates better analgesia on using fentanyl with levobupivacaine.

Our study was agreed with Eissa et al. ${ }^{(20)}$ who showed in their study carried on 90 patients of 18-60 years old with ASA physical status I/II scheduled for elective surgery of upper limb, they were divided into three groups to study effect of adding fentanyl and dexmedetomidine to $0.5 \%$ levobupivacaine. They showed that people receiving Fentanyl with levobupivacaine mixture have lower VAS score than control group.

Furthermore, our results agreed with manibr $\boldsymbol{e t}$ al. (21) who showed in their study comparing the analgesic efficacy of dexmedetomidine and fentanyl as adjuvants to levobupivacaine in supraclavicular 
brachial plexus block, in which pain scoring was assessed using 4-point verbal numeric rating pain scale (VNRS). they showed significant difference between control group and group receiving fentanyl additive.

Also, our study came in agreement with Kumar et $\boldsymbol{a l} .{ }^{(16)}$ they showed in their study on using dexmedetomidine and fentanyl as an adjuvant to levobupivacaine in supraclavicular brachial plexus block, using numerical pain scale ( 0 no pain / 10 most severe pain ) at thirty minutes, two hours, four hours, six hours, eight hours and at twelve hours.

The mean pain score was lower in patients receiving fentanyl than control group and there was significant difference.

As regard analgesic inquiry during the 24 hours postoperative, we found that $58 \%$ of patients in Group LF didn't ask for analgesia compared to $22 \%$ of patients in group L.

The first analgesic inquiry in Group LF $(372.8 \pm 29.3 \mathrm{~min})$ was prolonged compared to Group L $(279 \pm 44 \mathrm{~min})$. This showed that adding fentanyl prolongs the period of postoperative analgesia.

Our results also agreed with Hembrom et al. ${ }^{(13)}$ who showed in their study on the effects of adding Fentanyl to Levobupivacaine in supraclavicular brachial plexus block, which was carried out on 100 patients showed that adding fentanyl prolongs the postoperative analgesia time and prolongs the 1ST analgesic inquiry time $(1260 \pm 117 \mathrm{~min})$ compared to

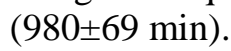

Shiva and Kumar (10) showed in their comparative study between bupivacaine alone and bupivacaine with fentanyl in axillary plexus block that the number of patients who required rescue analgesia and the mean number of supplemental analgesic boluses required were also significantly lower in patients receiving fentanyl additive.

Also, our results came in agreement with Farooq et al. (11) in their study to evaluate the efficacy of fentanyl and dexmedetomidine as adjuvant to ropivacaine in brachial plexus block carried out on 105 patients divided into 3 groups, group I fentanyl with ropivacaine, group II dexmedetomidine with ropivacaine, and group III ropivacaine with normal saline. They showed that fentanyl had a prolonged postoperative analgesia as compared to control groups and highly significant difference between both groups (Pvalue < 0.0001). Mean time for 1st analgesic inquiry was $7.54 \pm 0.51 \mathrm{~h}$ in fentanyl group as compared to 4.03 $\pm 0.75 \mathrm{~h}$ in control group.

Supraclavicular brachial plexus block is widely employed regional nerve block to provide anesthesia and analgesia for the upper extremity surgery. It provides rapid, dense and predictable anesthesia of the entire upper extremity. It is a practical alternative to general anesthesia for significant surgery on the upper limb providing a superior quality of analgesia and avoids the common side effects associated with general anesthesia. These blocks are therefore particularly useful in the ambulatory surgical setting for a wide variety of patients and procedures ${ }^{(16)}$.

We used Levobupivacaine, the isolated S-isomer of bupivacaine. it showed less cardiotoxicity than bupivacaine. It has same efficacy for the time to reach sensory block and total duration of sensory block as that of bupivacaine, but it has better toxicity profile than bupivacaine ${ }^{(22)}$.

As regard technical complications of supraclavicular brachial Plexus block such as hematoma and pneumothorax were not observed in our study. No respiratory depression was observed in any patient of the study. Hypotension and Bradycardia were noted in one patient in Group LF. Bradycardia occurred in one patient of Group L. Incidence of nausea was recorded in Group LF in two patients while in one patient in Group L.

Paluvadi and Sasha ${ }^{(8)}$ study on the effect of addition of fentanyl to xylocaine hydrochloride in supraclavicular brachial plexus block observed that there is incidence of nausea in $13.3 \%$ in patients receiving fentanyl with xylocaine and also vomiting in $6.6 \%$ in patients of the same group compared to zero $\%$ in patients of the study group.

Eissa et al. ${ }^{(20)}$ in their study on the Effect of adding dexmedetomidine or fentanyl to levobupivacaine for supraclavicular brachial plexus block carried on 50 patients, showed only one patient presented with nausea in the group receiving fentanyl and levobupivacaine mixture, no other complications were noted

Manbir et al. (21) they showed in their study comparing the analgesic efficacy of dexmedetomidine and fentanyl as adjuvants to levobupivacaine in supraclavicular brachial plexus block that no significant side effects were noted in any group.

\section{CONCLUSION}

It could be concluded that using fentanyl as an adjuvant to levobupivacaine is effective in enhancing the onset of the brachial plexus block and prolonging the duration of motor and sensory blocks with no hemodynamic changes. It effectively prolongs postoperative analgesia and lowers the analgesic requirements.

\section{REFERENCES}

1. Nael G, Gerancher J, Hebl J et al. (2009): Upper extremity regional anesthesia essentials of our current understanding. Regional Anesthesia Pain Med., 34: 134170.

2. Sanford M, Keating G (2010): Levobupivacaine: a review of its use in regional anesthesia and pain management. Drugs, 70: 761-791. 
3. Bone H, Van Aken H, Brooke M (2000): Enhancement of axillary brachial plexus block anesthesia by coadministration of neostigmine. Regional Anesthesia and Pain Med., 24: 405-415.

4. Rajkhowa T, Das N, Parua S (2016): Fentanyl as an adjuvant for brachial plexus block: A randomized comparative study. International Journal of Clinical Trials, 3: 64-67.

5. Munipalle S, Gonapa B (2017): Effect of Addition of fentanyl on the onset and duration of action of ropivacaine in brachial plexus block. IOSR Journal of Dental and Medical Sciences, 16: 109-112.

6. Chavan S, Koshire A, Prasad P (2011): Effect of addition of fentanyl to local anesthetic in brachial plexus block on duration of analgesia. Anesthesia Essays and Researches, 5: 39-46.

7. Kumar R, Kumar A, Verma $R$ et al. (2019): Comparison of Fentanyl and Nalbuphine as an Adjuvant to $0.5 \%$ Bupivacaine for Ultrasound Guided Supraclavicular Brachial Plexus Block. Journal of Clinical and Diagnostic Research, 13: 6-10.

8. Paluvadi V, Sasha V (2019): Effect of addition of fentanyl to xylocaine hydrochloride in brachial plexus block by supraclavicular approach. Anesthesia Essays and Research, 11: 121-124.

9. Hamed M, Ghaber S, Reda A (2019): Dexmedetomidine and fentanyl as an adjunct to bupivacaine $0.5 \%$ in supraclavicular nerve block randomized controlled study. Anesthesia Essays and Research, 12: 475-479.

10. Shiva P, Kumar A (2018): Comparative study between bupivacaine alone and bupivacaine with fentanyl in axillary brachial block. Journal of Evidence-Based Medicine, 5: 1886-1892.

11. Farooq N, Singh R, Sarkar A et al. (2017): evaluate the efficacy of fentanyl and dexmedetomidine as adjuvant to ropivacaine in brachial plexus block, Anesthesia Essays and Research, 11: 730-739.

12. Mcleod M, Burke D (2001): Levobupivacaine. Anesthesia, 56: 331-342.

13. Hembrom B, Kujur A (2018): A Study on Effects of Adding Fentanyl to Levobupivacaine (0.5\%) In
Supraclavicular Brachial Plexus Block. IOSR Journal of Dental and Medical Sciences, 3: 8-14.

14. Hassan S, Ahmed A, Syeda $\mathbf{N}$ et al. (2018): Efficacy and Safety of Fentanyl as An Adjuvant with Bupivacaine and Lignocaine in Supraclavicular Brachial Plexus Block. Chattagram Shishu Hospital Medical College Journal, 17: 31-39.

15. Manohar P, Parakash M (2015): Comparison of the Effects of Fentanyl and Dexmedetomidine in Supraclavicular Brachial Plexus Block Achieved with $0.5 \%$ Bupivacaine. Journal of Medical Science and Clinical Research, 3: 7131-7138.

16. Kumar A, Agrawal A, Sarafaraj A (2016): Comparison between fentanyl and dexmedetomidine as adjuvant to levobupivacaine in supraclavicular brachial plexus block. Anesthesia Essays and Research, 12: 669-673.

17. Shrestha B, Maharjan S, Shrestha S et al. (2007): Comparative study between tramadol and dexamethasone as an admixture to bupivacaine in supraclavicular brachial plexus block. Journal of Nepal Medical Association, 46: 158-164.

18. Karakaya D, Büyükgöz F (2001): Addition of fentanyl to bupivacaine prolongs anesthesia and analgesia in axillary brachial plexus block. Regional Anesthesia and Pain Med., 26: 434-438.

19. Fields H, Emson P, Leigh B et al. (1990): Multiple opiate receptor sites on primary afferent fibers. Nature, 284: 351-353.

20. Eissa R, Abo-Elnassr L, Amin S et al. (2018): The Effect of Adding Dexmedetomidine or Fentanyl to Levobupivacaine for Ultrasound-guided Supraclavicular brachial plexus block in Upper Extremity Surgeries. The Egyptian Journal of Hospital Medicine, 73: 6004-6009.

21. Manibr K, Rupinder K, Baghla N et al. (2018): Study to Compare the Analgesic Efficacy of Dexmedetomidine and Fentanyl as Adjuvants to Levobupivacaine in Ultrasound-Guided Supraclavicular Brachial Plexus Block. Anesthesia Essays and Research, 12: 669-673.

22. Leone S, Di Cianni S, Casati A et al. (2008): Pharmacology, toxicology, and clinical use of new long acting local anesthetics, ropivacaine and levobupivacaine. Acta Bio Medica, 79: 92-105. 